\title{
Efficacy of a Transdiagnostic internet-based treatment for emotional disorders with a specific component to address positive affect: Study protocol for a randomized controlled trial
}

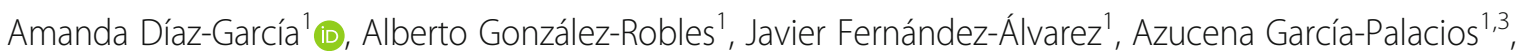 \\ Rosa María Baños ${ }^{2,3}$ and Cristina Botella ${ }^{1,3^{*}}$
}

\begin{abstract}
Background: Emotional disorders (ED) are among the most prevalent mental disorders. However, less than 50\% of people suffering from ED receive the appropriate treatment. This situation has led to the development of new intervention proposals based on the transdiagnostic perspective, which tries to address the underlying processes common to ED. Most of these programs focus primarily on down-regulating negative affectivity, rather than increasing strengths and up-regulating positive affectivity. The data suggest the existence of disturbances in positive affectivity in these disorders, and so new interventions focusing on these problems are greatly needed. It is also essential to provide assistance to all the people in need. Information and Communication Technologies can be very useful. This study aims to evaluate the efficacy of a transdiagnostic Internetbased treatment for ED in a community sample. The protocol includes traditional CBT components, as well as a specific component to address positive affect. We intend to test this protocol, including this specific component or not, versus a waiting list control group. Moreover, we aim to test the differential effect of this specific component, and study the effectiveness (in terms of patients' acceptance) of using a self-applied Internet-based program. This paper presents the study protocol.
\end{abstract}

Methods: The study is a randomized controlled trial. 207 participants will be randomly assigned to: a) Transdiagnostic Internet-based protocol (TIBP), b)Transdiagnostic Internet-based protocol + positive affect component $(T I B P+P A)$, or c)a Waiting List control group (WL). Primary outcomes measures will be the BDI-II, the BAI, and the PANAS. Secondary outcomes will include diagnosis-specific measures of the principal disorder. Participants' treatment acceptance will also be measured. Participants will be assessed at pre-, post-treatment, and 3- and 12- month followups. The data will be analyzed based on the Intention-to-treat principle. Per protocol analyses will also be performed.

Discussion: To the best of our knowledge, this is the first study of a transdiagnostic Internet-based treatment for ED with a specific component to up-regulate positive affectivity. This intervention could contribute to improve the efficiency and effectiveness of current treatment programs for ED, promote the dissemination of EBTs, and help to decrease the high prevalence of ED.

Trial registration: ClinicalTrial.gov: NCT02578758. Registered 15 October 2015.

Keywords: Transdiagnostic, Internet, Positive affectivity, Emotional disorders, Depression, Anxiety, Emotion regulation, Randomized controlled trial

\footnotetext{
*Correspondence: botella@uji.es

${ }^{1}$ Universitat Jaume I, Castellón, Spain

${ }^{3}$ CIBER Fisiopatología Obesidad y Nutrición (CIBERObn), Instituto Salud Carlos

III, Madrid, Spain

Full list of author information is available at the end of the article
} 


\section{Background} Introduction

Lifetime prevalence estimates for emotional disorders (ED), defined as anxiety and unipolar mood disorders, are quite high (anxiety disorders, 28.8\%; mood disorders, $20.8 \%$ ), with comorbidity rates ranging between 40 and $80 \%$ [1]. These disorders disrupt the lives of millions of people each year, and they are one of the main causes of disability worldwide [2, 3].

In the past few decades, evidence-based psychological treatments (EBTs) have been shown to be effective in the treatment of ED [4]. This development can be considered a significant advance in addressing the large worldwide treatment needs [5]. However, the scale of these treatments is not sufficient to reduce the disease burden of mental disorders [6]. Less than $50 \%$ of people suffering from ED receive adequate treatment [7], and this percentage is much lower in adolescents, older adults, people with a lower socio-economic status, and people from ethnic minorities [8].

These low levels of successful dissemination of EBTs can be explained by their costs, the duration of the treatments, and the lack of well-qualified professionals [9]. This is especially problematic in everyday clinical practice and can explain why EBTs are under-utilized [10]. It is not surprising, therefore, that this continued lack of widespread availability of EBTs has raised the need to implement innovative and even radical solutions to ensure that the aid reaches all those in need $[8,11,12]$. There is a compelling need for approaches that go beyond "one-to-one" psychotherapy (either with a patient, a family, or a group) and develop a new portfolio to administer EBTs [6].

Different EBTs targeting specific anxiety and mood disorders have been developed [13-16]. Each diagnosisspecific treatment manual requires the use of separate handbooks, workbook, and protocols, and therapists must be trained in the use of each of them, which may hinder widespread dissemination of EBTs [9].

In recent years, new proposals have emerged for transdiagnostic treatments that emphasize the essential processes underlying different disorders. In the field of ED, studies emphasize that these disorders share important characteristics, and that this overlap emerges from common biological and psychological vulnerabilities $[14,17,18]$. Barlow summarizes the commonalities in the etiology of ED in the model referred to as "triple vulnerabilities". This theory encompasses a generalized biological vulnerability (involving nonspecific genetic contributions to the development of anxiety and negative affect), a generalized psychological vulnerability (associated with early life experiences under certain conditions), and a specific psychological vulnerability emerging from early learning [14]. There is evidence that the two generalized vulnerabilities are involved in the development and expression of the ED $[18,19]$.

People with ED have higher levels of neuroticism/ negative affect/behavioral inhibition (N/NA/BI) [18], and they experience negative emotions more intensely and frequently [20,21], accept emotional experiences to a lesser extent [22], associate the experience of living with more negative emotions [23], use cognitive and behavioral strategies to reduce the impact of negative emotions [24], and show intolerance to uncertainty, leading to an increase in negative affect [25]. In short, people with ED tend to react negatively to their emotions and are more likely to use maladaptive emotion regulation strategies. These strategies, in turn, increase the frequency/intensity of negative emotions. Some authors have argued that this functional relationship may be driven by neuroticism, which would be the core of the ED [26].

By contrast, the role of extraversion/positive affect/behavioral activation (E/PA/BA) in ED has also been pointed out. A meta-analysis indicated that most individuals with an ED show low levels of E/BA [27]. The data suggest that alterations in PA are observed in many disorders [28]. Low levels of PA predict the onset of depression [29], dampen positive emotions, are maladaptive, and increase the severity of the problem [30], whereas high PA is associated with better health, both physical and psychological, and greater well-being [31]. Despite the importance of disturbances in positive affectivity in ED, few studies focus on promoting PA.

Based on the transdiagnostic perspective, Barlow's team designed the Unified Protocol (UP): a transdiagnostic, emotion-focused, cognitive-behavioral treatment for ED that emphasizes the role of emotion regulation $[9,26,32]$. The UP focuses on four essential aspects that have the general purpose of down-regulating NA: addressing emotional avoidance, promoting cognitive flexibility, and facilitating exposure to avoided situations and sensations. Moreover, it places special emphasis on increasing present-focused emotional awareness. The UP has been tested, and results indicate that it is effective [33], with improvements maintained at 18-month follow-up [34]. Moreover, the effect of the UP has been shown on the two temperament dimensions of N/BI and E/BA [35].

The data suggest that a transdiagnostic treatment for ED might be more widely effective across diverse mental health problems, in other words, treatments aimed at addressing different disorders with a single protocol [36]. Some meta-analyses have been conducted on the efficacy of transdiagnostic protocols for anxiety disorders [37] and for anxiety and/or depression [38-40].

Nevertheless, these transdiagnostic protocols have focused on reducing NA, but less attention has been paid 
to promoting PA or modifying risk factors. As the World Health Organization's definition of mental health expresses, mental health is more than just the absence of mental illness [41]. It is not surprising, therefore, that well-being and positive functioning are considered core elements of mental health. As Southwick and Charney [42] point out, the use of procedures to promote resilience (such as positive emotions and optimism) can be useful in the treating ED and in generating protective factors. The benefits may be associated with a reduction in the risk of developing mental symptoms and disorders $[43,44]$. However, as stated above, interventions that include components to up-regulate PA have been missing or very scarce in the clinical setting.

Taking all this into consideration, it is necessary to develop and test treatment components focused on enhancing protective factors and resilience and mitigating risk factors. Literature has highlighted the potential importance of positive emotionality as a treatment component $[45,46]$.

To date, the dominant delivery format in psychotherapy has been individual face-to-face contact; however, it is much more expensive and time-consuming than other formats, such as guided self-help and Internet-based treatments [47]. Recently, research has shown that Information and Communication Technologies (ICT) can facilitate the availability of EBTs [6, 48, 49]. Specifically, some literature suggests that the Internet can be used for the assessment and treatment of clinical conditions [50]. Internet-based treatments are interventions conducted over the Internet with more or less therapist involvement and support [51]. The evidence strongly suggests that Internet-based treatments are effective in the treatment of depression and anxiety disorders [52]. Moreover, data from meta-analyses reveal that these interventions are as efficacious as face-to-face traditional treatments [53-55].

We have developed an online psychological treatment protocol for individuals with a diagnosis of ED [56], major depression disorder (MDD), dysthymic disorder (DD), obsessive-compulsive disorder (OCD), and four anxiety disorders: panic disorder (PD), agoraphobia (AG), generalized anxiety disorder (GAD), social anxiety disorder (SAD), anxiety disorder not otherwise specified (ADNOS), and (unipolar) mood disorder not otherwise specified (MDNOS).

This treatment protocol includes two types of components: one based on classical perspectives for downregulating NA and the other aimed at up-regulating PA. The protocol can be applied either in its traditional format (Transdiagnostic Internet-based Protocol -TIBP-) or by including both of these components (Transdiagnostic Internet-based Protocol + Positive Affect Component TIBP + PA-). Moreover, we have developed an adaptation of the treatment protocol that can be applied online over the Internet. We can thus reach community samples, that is, people who suffer from an ED but are not receiving primary or specialized care.

We intend to study the effect of both treatments in terms of efficacy for depressive and anxious symptomatology. Moreover, we aim to assess the effects of the specific treatment component designed for up-regulating PA. Finally, the effectiveness of the Internet-based program developed to apply the treatment protocol over the Internet with minimal support by the clinician will be studied. We hypothesize that: a) both self-applied protocol modalities $(T I B P$ and $T I B P+P A)$ will be more effective than the waiting list control condition in the treatment of ED; b) both interventions will result in significant improvements in depressive and anxious symptomatology at post- treatment, and these results will be maintained at 3- and 12- month follow-ups; c) the TIBP + PA will significantly outperform the TIBP group on PA measures; and d) both protocols will be well accepted, with no statistical differences between conditions. In this article, we present the study protocol.

\section{Methods/Design \\ Study design}

A three-armed randomized controlled trial (RCT) will be conducted. Participants will be randomly allocated to one of three conditions: a) Transdiagnostic Internet-based protocol (TIBP), b) Transdiagnostic Internet-based Protocol + Positive Affect component $(T I B P+P A)$, and c) Waiting List control condition (WL). For ethical reasons, participants in the control condition will be offered the possibility of receiving the treatment protocol. Block randomization will be performed within each stratum in order to ensure that all primary diagnoses are equally represented across conditions. Measures will be taken at post-randomization, after the treatment, and at 3- and 12-month follow-ups, in order to test whether the improvements achieved during the therapy are maintained in the long term.The study flowchart is shown in Fig. 1. The study will be conducted following the CONSORT statement (Consolidated Standards of Reporting Trials, http://www.consort-statement.org) [57, 58] the CONSORT-EHEALTH guidelines [59], and the SPIRIT guidelines (Standard Protocol Items: Recommendations for Interventional Trials) $[60,61]$.

\section{Sample size}

To determine the sample size, the effect sizes found in the literature have been considered. An RCT using the UP in a traditional face-to-face approach [33] obtained effect sizes of 0.56 for anxiety, measured with the BAI, and 1.11 for depression, measured with BDIII. The mean effect size indicated in a recent metaanalysis [39] comparing transdiagnostic computerized 


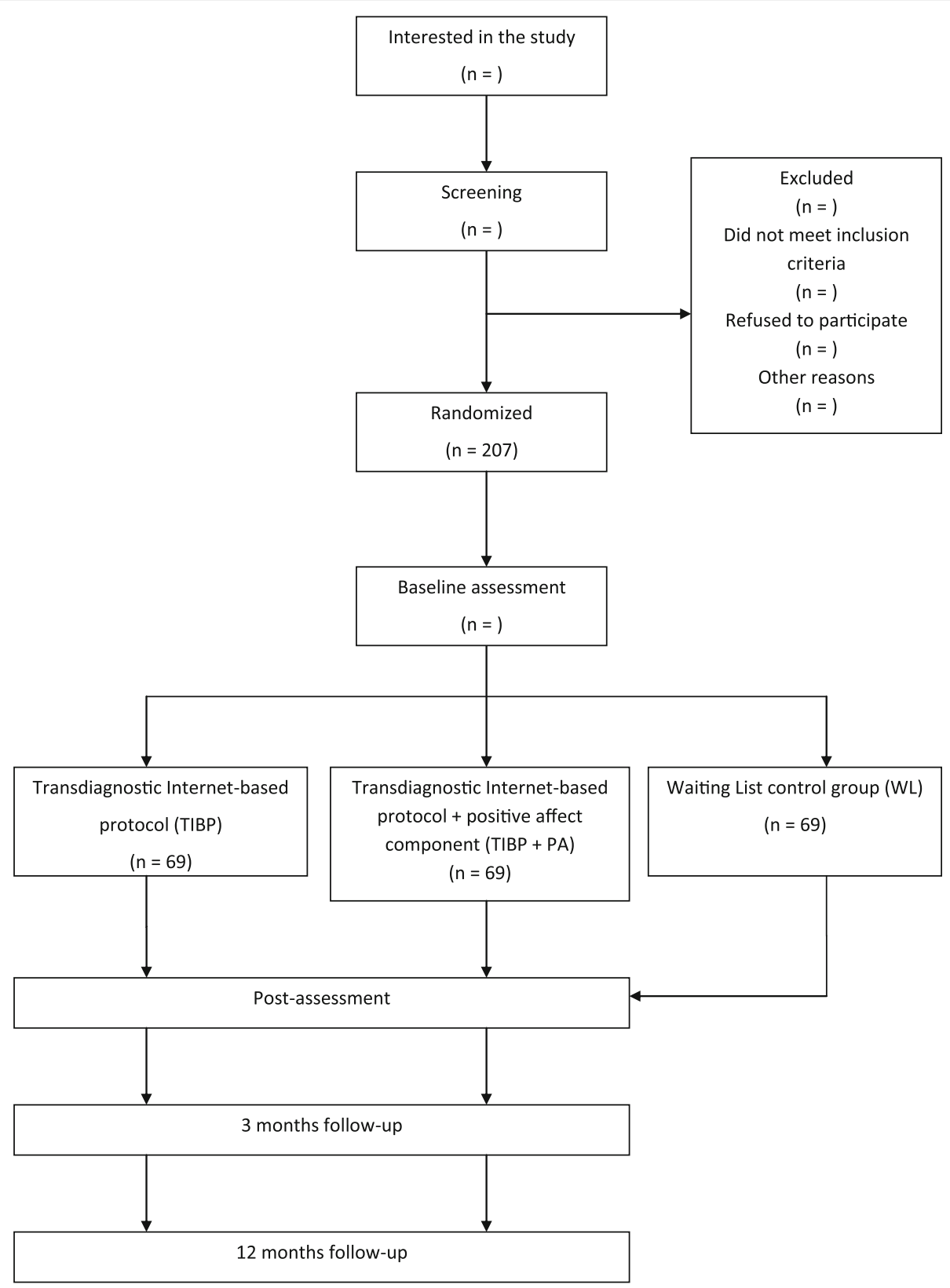

Fig. 1 Flowchart of participants

cognitive-behavior therapy (CBT) to waitlist control was $g=.93$ for anxiety and $g=.88$ for depression.

Adopting a more conservative approach than those of the aforementioned studies, we assumed an effect size of 0.5 (Hedges' g), which, following Cohen [62], can be considered an effect size of medium magnitude. As our design included three experimental conditions (TIBP, TIBP $+P A, W L$ ), a between-groups one-way ANOVA was assumed for the statistical analyses. Therefore, calculations of the necessary sample size cannot be based on Hedges' $g$ index for effect size, but rather on the $f$ index [62]. Following Cohen
[62], $f=0.25$ represents an effect size of medium magnitude (and equivalent to $g=0.5$ ). Thus, considering an alpha of .05 and a statistical power of .80 , the total sample size required to warrant these conditions contains 159 participants (53 participants per group). To control the maximum possible loss of subjects during treatment, based on the literature about Internet-based treatments, a $30 \%$ dropout rate is expected [53, 63]. Thus, the required sample size should have 207 participants in all (69 participants per group). These calculations were accomplished with the software program G*Power 3.1 [64]. 


\section{Study population, recruitment and eligibility criteria}

The clinical trial will be conducted in a community sample of patients diagnosed with the aforementioned disorders. Participants will be adult volunteers who contact us because they are interested in the study (personal visits or phone calls to the emotional disorders university clinic, emails, or leaving their data on a website specially prepared for this purpose). Potential participants will be attended to by a psychologist who will describe the study characteristics to them. All the psychologists working on this study will have at least a master's degree in Clinical Psychology, with experience in the diagnosis, psychological assessment, and application of EBTs for ED.

The psychologist will clarify any doubts, ensure that the participant has read the information about the study, and make sure that $\mathrm{s}$ /he has understood the three experimental conditions. People interested in participating will sign an online informed consent and be assessed taking into account all the inclusion criteria. Inclusion and exclusion criteria are shown in Table 1 . If the patient fulfills all the study criteria, the researcher will contact an independent researcher to implement randomization. This researcher will be unaware of the characteristics of the study. Randomization will be performed using weighted random allocation [65] in order to take into account the clinical features (different diagnoses) and, thus, obtain a homogeneous distribution in the three experimental conditions. Participants agree to participate before finding out to which treatment they will be allocated. All participants will be free to withdraw from the treatment at any time.

\section{Ethics}

The study follows the guidelines of the Declaration Helsinki and existing guidelines in Spain and the European Union for the protection of patients in clinical trials. As noted, all participants will be volunteers, and they will sign the online informed consent to form part of the study once it has been explained to them.

Table 1 Inclusion and exclusion criteria

\begin{tabular}{|c|c|}
\hline Inclusion criteria & Exclusion criteria \\
\hline Minimal age of 18 years & $\begin{array}{l}\text { Suffering from Schizophrenia, bipolar } \\
\text { disorder, or alcohol and/or substance } \\
\text { dependence disorder }\end{array}$ \\
\hline $\begin{array}{l}\text { Meeting the DSM-IV diagnostic } \\
\text { criteria for ED }\end{array}$ & High risk of suicide \\
\hline $\begin{array}{l}\text { Ability to understand and read } \\
\text { Spanish }\end{array}$ & $\begin{array}{l}\text { Medical disease/condition that } \\
\text { prevents the participant from carrying } \\
\text { out the psychological treatment }\end{array}$ \\
\hline $\begin{array}{l}\text { Access to Internet at home and } \\
\text { having an email address }\end{array}$ & $\begin{array}{l}\text { Receiving another psychological } \\
\text { treatment during the study }\end{array}$ \\
\hline $\begin{array}{l}\text { Providing online informed } \\
\text { consent }\end{array}$ & $\begin{array}{l}\text { An increase and/or change in the } \\
\text { pharmacological treatment (in the } \\
\text { case of receiving) during the study } \\
\text { period }\end{array}$ \\
\hline
\end{tabular}

Participants will have the possibility of withdrawing from the study at any time. The recruitment of participants will be carried out by qualified personnel from a clinical point of view. Participants allocated to the $W L$ condition will be offered the opportunity to receive the TIBP or the $T I B P+P A$ intervention after the waiting time has ended. Based on the literature, no special difficulties are expected. However, any undesired event would not only mean the participant's departure from the trial, but $\mathrm{s} /$ he would also be offered the possibility of receiving psychological care at the Emotional Disorder Clinic in Universitat Jaume I, or of being referred if his/her medical condition required it.

A fundamental aspect in a project of this nature is data protection. To protect information, strategies using personal passwords and data via AES encryption (AES256; Advanced Encryption Standard) will be used. Personal data will be replaced by codes and data, which must be collected by clinicians (e.g. age, sex, address, and phone), stored separately from other data, and only made available to researchers responsible for the study, always protecting the right to privacy.

The study has been approved by the Ethics Committee of Universitat Jaume I (Castellón, Spain). The trial was registered at clinicalstrial.gov as NCT02578758.

\section{Interventions}

We have developed a manualized protocol, based on the transdiagnostic perspective for the treatment of ED: MDD, DD, OCD, PD, AG, GAD, SAD, ADNOS and MDNOS. The treatment protocol is structured in a patient handbook and a therapist handbook (Botella C, García-Palacios A, Quero S, Baños R. A Transdiagnostic Treatment for Emotion Disorders: Manualized Treatment Protocol, unpublished). The modules in each intervention protocol are described briefly in the following section.

We have adapted this protocol from classic perspectives derived from the UP $[9,17]$ and some strategies from Marsha Linehan's protocol [66]. The protocol includes the following core components mainly addressed to downregulate NA: present-focused emotional awareness and acceptance, cognitive flexibility, behavioral and emotional avoidance patterns, and interoceptive and situational exposure. The protocol also includes traditional therapeutic components of evidence-based treatment for ED (Psychoeducation, Motivation for change, and Relapse prevention). In addition, in order to promote psychological strengths and enhance well-being [67], we have included a PA-regulation component based on behavioral activation strategies [68], strategies to promote pleasant and significant activities linked to values and life goals, and strategies to enhance personal strengths, positive feelings, positive cognitions, and positive behavior $[67,69]$. This component also includes the Well-being Therapy (WBT) [70, 71] 
strategy of identifying examples of well-being and negative cognitions ("interrupting thoughts") that interfere with these moments of well-being, in order to modify these interfering cognitions. Some notions of Fredickson's Broaden-and-Build Theory are also included to explain the mechanisms behind positive emotions [31].

\section{Transdiagnostic Internet-based protocol (TIBP)}

This intervention protocol consists of twelve more traditional modules mainly designed to down-regulate NA. Each module has specific objectives:

\section{M1. Emotional disorders and emotion regulation. The} purpose of this module is to provide information about the central role of emotion regulation in emotional disorders. Brief descriptions of the program modules, as well as videos with examples of people suffering from different ED, are also presented.

M2. Motivation for change. The objective of this module is to enhance motivation for change by recognizing that attitudes towards change can be ambivalent and that motivation fluctuates. The main objective is to analyze the advantages and disadvantages of changing, emphasize the importance of being motivated, and highlight the importance of establishing significant life goals.

M3. Understanding the role of emotions. This module provides information about the adaptive roles and functions of emotions. It also shows the threecomponent model of emotions.

M4. The acceptance of emotional experiences. This module focuses on the awareness and role of acceptance of emotional experiences, as well as their importance in the treatment.

M5. Practicing acceptance. The objective of this module is to continue to learn about the acceptance of emotional experiences and increase awareness of physical sensations, thoughts, emotions, and daily activities.

M6. Learning to be flexible. This module teaches participants to be more cognitively flexible in order to see life situations from different perspectives, showing the importance of maladaptive ways of thinking and learning how to identify them in the maintenance of emotional disorders.

M7. Practicing cognitive flexibility. This module aims to teach the patients how to modify maladaptive ways of thinking. It also provides information about intrusive thoughts and how to deal with them.

M8. Emotional avoidance. The aim is to teach the patients emotion avoidance strategies that contribute to the maintenance of emotional disorders.

M9. Emotion Driven Behaviors (EDBs). The aim is for patients to learn the concept of EDBs and replace their own maladaptive EDBs with other more adaptive behaviors.

M10. Accepting and facing physical sensations. The objectives are to teach the patients the role of physical sensations in their emotional response and train them in interoceptive exposure, in order to increase tolerance and promote habituation to physical sensations. M11. Facing emotions in the contexts where they occur. This module aims to increase tolerance to emotions and reduce avoidance behavior. The purpose is to construct exposure hierarchies to help patients to begin to face the avoided situations that contribute to the maintenance of the problem.

\section{Transdiagnostic Internet-based protocol + positive affect component (TIBP + PA)}

This intervention protocol is made up of 16 therapeutic modules. The first 12 modules have been described above in the first condition - (TIBP) - and the other 4 are aimed to up-regulate PA by understanding and capturing positive emotions, and training adaptive positive emotion regulation strategies. These four modules are described as follows:

M12. Learning to move on. This module focuses on the role of behavioral activation. The aim is to teach the importance of 'moving on' in acquiring a proper level of activity and involvement in life. It motivates the patient to get involved in meaningful activities and become engaged in his/her life.

M13. Learning to enjoy. This module helps the patient to see the importance of positive emotions and teaches procedures that generate positive experiences, promoting involvement in pleasant and significant activities and contact with others. This module involves enjoying positive experiences and "savoring" positive aspects of life in order to enhance wellbeing.

M14. Learning to live. This module takes a further step in enhancing PA, understanding the importance of identifying the individual's own psychological strengths, and selecting and carrying out meaningful activities linked to values and goals in life. This module provides strategies to achieve psychological well-being and resilience. The aim is to improve the individual' abilities and live a life full of purpose and meaning.

M15. Living and learning. This module focuses on developing an action plan to boost the individual's psychological strengths. It focuses on the importance of developing and enhancing one's own strengths and starting to work for life and the future.

M16. Relapse prevention (this module is the same for both conditions: (TIBP) and (TIBP + PA). It aims to strengthen the strategies learned throughout the program, schedule future practice, and teach how to identify and cope with future high- risk situations. 


\section{Adaptation to the web}

This protocol has been adapted to a multimedia web platform (video, images, etc.) and can be applied over the Internet (https://www.psicologiaytecnologia.com/). It is developed for optimal use on a PC or a tablet, and it allows individuals to do the modules from their home and at their own pace. The ease of use of the program has been strengthened because it presents a streamlined navigation, which allows users with less experience in handling new technologies to know where they are and how to keep moving forward at any time. The web adaptation of the protocol is the same in both conditions (TIBP and TIBP + PA), with the only difference being the inclusion or not of the modules that contain the PAregulation component.

Duration of the program can vary among users, and participants in both treatment conditions have access to the protocol for a maximum period of 18 weeks. The program recommends working on one module for at least one entire week, and it sends messages encouraging the patient to continue to work to benefit from it. In both conditions, the modules include exercises and tasks to practice each technique and skill. Moreover, the platform is ready to welcome all participants with information about the treatment and its objectives, as well as general information and recommendations about how to benefit from it.

Regarding the assessment of patients within the program, after the "Welcome", initial online questionnaires are presented as the pre-treatment assessment. In addition, post-module brief questionnaires to evaluate anxiety, depression, and positive/negative affect (OASIS, ODSIS, PANAS) are also filled out throughout the program. A noteworthy aspect of the program is that it allows the therapist to have access to all this information and receive an alert if the patient's condition worsens. The program includes suicide risk alarms that consist of an email to the clinical team with information about a high risk of suicide. These alarms are generated when the system detects that the participant rated high on the suicidal ideation items. It allows the clinician to contact the patient and evaluate the actions that should be taken to protect him/her. Finally, after the treatment, the participants complete the online assessment in the postand follow-up periods.

All the modules are sequential, which allows the participant to move through the program step by step. The web platform was designed to optimize the understanding of the modules' content. It has different multimedia elements (vignettes, videos, audios,...) that help the user to assimilate the different psychological techniques in the easiest way. The modules always follow the same structure: they start with questions related to the previous module, continue with the specific contents of the module, then propose the exercises, and finally present a series of simple self-assessment questions designed to verify whether the content presented has been understood correctly. In addition, at the end of each module, homework tasks are indicated to work on what was presented in the module. Performing these tasks helps to consolidate everything learned in the program. Participants have the opportunity to obtain printable documents (PDF) with summaries of each module.

The web platform has four complementary tools that appear on the main menu of the protocol: 1) "Home", which is the starting point from which the participant can access the other sections of the protocol; 2) "Calendar", which is a tool that allows the participant to know about pending or achieved tasks as well as his/her current point in the program; 3) "Review", which allows the participant to review the treatment modules already completed; and 4) "How am I?", which allows participants to monitor their progress through a set of graphs. It is a tool that provides feedback to participants about their evolution during the program with regard to their emotional distress (anxiety and sadness) and their positive and negative affectivity.

\section{Support}

In each of the two treatment conditions, we will provide human support and ICT support to all participants.

Human support will be provided by weekly phone calls (maximum of $5 \mathrm{~min}$ ) during the treatment period in order to resolve any difficulties or doubts about the use of the online protocol, or to remind them of the importance of doing the homework tasks and reviewing the treatment contents. During these weekly phone calls, we will also encourage participants to continue to use the protocol, and reinforce them for engaging in the treatment. At the end of the treatment period, participants will be reminded that they will be contacted again for follow-up assessments.

ICT support will consist of two weekly mobile phone text messages reminding participants of the importance of reviewing the modules and encouraging them to do the homework tasks. Some examples of these text messages are: "Hi there! Don't hesitate to review the modules you've already completed if necessary. Remember, practice makes perfect!"or "Hi there! Don't give up on your module tasks! Dedicate some time and effort to them. Remember, it's ideal to complete one module each week". These messages will be randomized, so that participants do not always receive the same content. A professional platform will be used to send these messages (www.trendoo.es). The online treatment also contains several multiplechoice questions about the contents seen in each module. The program immediately provides the 
participant with the correct feedback and a simple explanation. This aspect is repeated throughout all the treatment modules. In addition, the ICT support includes automatic e-mails with reminders to access the program when participants have not entered in the past 15 days.

\section{Measures}

\section{Diagnostic interview}

Mini International Neuropsychiatric Interview Version 5.0.0 (MINI) [72]. It is a short, structured, diagnostic psychiatric interview for DSM-IV and ICD-10 diagnoses. The MINI can be used by clinicians after a brief training session. Nonclinical interviewers should receive more intensive training. This interview has excellent interrater reliability $(\mathrm{k}=.88-1.00)$ and adequate concurrent validity with the Composite International Diagnostic Interview [73]. The MINI has been translated and validated in Spanish [74].

\section{Primary outcomes measures}

Beck Depression Inventory (BDI-II) [75] It is one of the most widely-used questionnaires in the evaluation of depression severity. The BDI consists of 21 items, scored on a scale from 0 to 3 , covering all the different symptoms of major depression disorder. The total score on this questionnaire can yield a maximum of 63 points. The instrument has shown good internal consistency $(\alpha=0.76$ to 0.95$)$. The Spanish version of this instrument has also shown high internal consistency $(\alpha=0.87)$ for both general and clinical populations $(\alpha=.89)[76]$.

Beck Anxiety Inventory (BAI) [77] The BAI is a 21item symptom checklist designed to assess anxiety symptoms. Each item is scored on a 0 to 3 point severity scale, and the total scores range between 0 and 63 . The internal consistency of the BAI has been found to range from .85 to .94. The Spanish version of the BAI has shown high internal consistency $(\alpha=.93)$ [78].

Positive and Negative Affect Scale (PANAS) [79] The PANAS consists of 20 items with a range from 1 (very slightly or not at all) to 5 (extremely). Respondents have to indicate the extent to which they experienced the feeling or emotion during the past few weeks. This scale evaluates two independent dimensions: PA and NA. The maximum score for each subscale is 50 . The scale showed excellent internal consistency ( $\alpha$ between .84 and .90) and convergent and discriminant validity. The Spanish version has demonstrated high internal consistency $(\alpha=0.89$ and 0.91 for PA and NA in women, respectively, and $\alpha=0.87$ and 0.89 for PA and $\mathrm{NA}$ in men, respectively) in college students [80].

\section{Secondary outcomes measures Diagnosis-specific measures}

Depending on each participant's main diagnosis, different instruments will be implemented.

- OCD: Obsessive-Compulsive Inventory-Revised (OCI-R) [81]. The OCI- $\mathrm{R}$ is a short scale made up of 18 items rated from 1 to 4 to assess obsessivecompulsive symptoms. The OCI-R yields six subscales: washing, checking, ordering, obsessing, hoarding, and neutralizing. The OCI-R has good internal consistency ( $\alpha=.81$ to .93 ), good to excellent test-retest reliability ( $\alpha=.57$ to .91 ), good convergent validity, and a solid factor structure. The Spanish version of the OCI-R has been found to be $\operatorname{good}(\alpha=.86)$ [82].

- PD/AG: Self-Reported Panic Disorder Severity Scale (PDSS-SR) [83]. The PDSS-SR is a 7-item self-report measure of panic disorder severity. This scale assesses panic attack frequency, distress during panic attacks, severity of anticipatory anxiety, fear and avoidance of agoraphobic situations, fear and avoidance of physical sensations, and work and social impairment. The scale has shown excellent reliability $(\alpha=.917)$, test-retest reliability (ICC $=.81)$, and sensitivity to change. The psychometric analysis of the Spanish version showed excellent internal consistency $(\alpha=.85)$, good testretest reliability, and adequate convergent validity [82].

- GAD: Penn State Worry Questionnaire (PSWQ) [84]. It is a questionnaire that evaluates symptoms related to GAD. The PSWQ is a 16-item self-report questionnaire that assesses the tendency to worry, as well as the intensity of the worry characteristic of GAD as an uncontrollable, generalized, and excessive experience. The PSWQ has demonstrated good internal consistency ranging from .91 to .95 , and good validity and test-retest reliability. The Spanish version of the scale showed an internal consistency of .90 and a testretest reliability of .82, as well as adequate convergent and discriminant validity [85].

- SAD: Social Interaction Anxiety Scale (SIAS) [86]. This scale is a 20-item self-report measure rated on a 5-point scale ranging from 0 (not at all characteristic or true of me) to 4 (extremely characteristic of me). It assesses cognitive, affective, and behavioral reactions in interactive social situations (symptoms related to social phobia). The SIAS has high internal consistency $(\alpha=.88$ to .94$)$ and good test-retest and discriminant reliability, as well as adequate construct validity. The Spanish validation of the scale showed adequate internal consistency [87]. 


\section{Personality measures}

NEO-five factor Inventory The NEO FFI is the short version of the NEO-PI-R [88], designed to assess the five personality dimensions through 60 items. In this study, only the subscales of neuroticism and extraversion are used. Each scale contained 12 items with a five-point Likert response format. Two-week retest reliability is uniformly high, ranging from 0.86 to 0.90 for the five scales [89], and internal consistency ranges from 0.68 to 0.86 [89].The Spanish version of the NEO FFI has been found to be good [90].

\section{Quality of life}

EuroQol 5D (EQ-5D-Spanish version) [91] is a generic instrument for measuring health-related quality of life. It can be used in relatively healthy individuals (general population) as well as in groups of patients with different pathologies. Each individual rates his/her own health on each of the two parts of the questionnaire. In the first part, the individual must check the level corresponding to his/her state of health in each of the five domains: mobility, self-care, daily activities, pain/discomfort and anxiety/depression. Each dimension is divided into three levels of severity (without problems, some problems or moderate problems, and severe problems), yielding a population-based preference score or societal index (SI). There are 243 possible combinations - health states -, and the SI is calculated on the basis of these health states. The index value ranges from 1 (best health state) to 0 (death), although there are negative values for the index corresponding to those health states that are rated as worse than death. In the second part of the questionnaire, the individual values his/her own health on a more general visual analogical scale (VAS), a $10 \mathrm{~cm}$ vertical line on which the best and worst imaginable health states score 100 and 0 , respectively.

\section{Suicidal ideation}

A suicide item has been included within the ODSIS [92] with the aim of obtaining a suicide risk indicator throughout the treatment. The total score depends on this single item, and the maximum score is 4 , with a range from 0 ("absence of thoughts of suicide") to 4 ("thoughts of suicide all the time").

\section{Post-module measures}

Scores on anxious and depressive symptomatology will also be obtained after each module has been completed.

Overall Anxiety Severity and Impairment Scale (OASIS) [93] The OASIS consists of a 5-item questionnaire with a scale from 0 to 4 , which measures the frequency and severity of anxiety, as well as the level of avoidance, work/academic/home interference, and social and everyday life impairment related to anxiety symptoms. The instructions tell the respondent to consider a wide range of anxiety symptoms (e.g., panic attacks, worries, flashbacks) when answering the questions, and the time frame is "during the past week". A psychometric analysis of the OASIS scale found good internal consistency $(\alpha=.80)$, test-retest reliability $(\mathrm{k}=.82)$ and convergent validity for this instrument. The Spanish version of the OASIS confirmed the factorial structure and reliability and validity data obtained by the original authors (internal consistency in both populations, general and clinical $(\alpha=.0 .86$ and test-retest reliability $(k=.84)$ [94].

Overall Depression Severity and Impairment Scale (ODSIS) [92] The ODSIS is a self-report measure with five items. Individuals select among five different response options ranging from 0 to 4 for each item. This scale evaluates experiences related to depression. The ODSIS measures the frequency and severity of depression, as well as the level of avoidance, work/academic/ home interference, and social and everyday life impairment related to depression symptoms. The ODSIS can also be used to assess severity and impairment associated with low mood. In the Spanish version of the ODSIS, the internal consistency has been shown to be excellent, with a Cronbach's alpha between .91 and .94 and good convergent and discriminant validity [95].

\section{Expectation of treatment scale and opinion of treatment scale.}

The Expectation of Treatment Scale and Opinion of Treatment Scale are questionnaires adapted from Borkovec and Nau [96]. Each scale contains five items, rated from 0 ("strongly disagree") to 10 ("strongly agree"), which cover how logical the treatment seemed, to what extent it could satisfy the patient, whether it could be used to treat other psychological problems, and its usefulness for the patient's specific problem. The expectation scale is applied once the intervention has been explained, at the end of the "Welcome module". This scale measures the patient's subjective expectations about the treatment. In addition, the opinion scale is administered when the patient has completed the treatment, and its aim is to assess satisfaction with the intervention. Our group has used this questionnaire in several research studies [48, 97].

The study measures and assessment times are summarized in Table 2 .

\section{Data analyses}

Intention-to -treat analyses and per protocol analyses will be performed, and CONSORT recommendations will be followed [98]. First, the three groups will be 
Table 2 Study measures and assessment times

\begin{tabular}{lll}
\hline Measure & Aim & Time of assessment \\
\hline MINI Neuropsychiatric Interview & Diagnosis & BL, Post-T and FU \\
PANAS & Positive and negative affect & Post-T and Post-module \\
BDI-II & Severity of depression & BL, Post-T and FU \\
BAI & Severity of anxiety & BL, Post-T and FU \\
OCI-R & Severity of OCD symptoms & BL, Post-T and FU \\
PDSS-SR & Severity of PD and & BL, Post-T and FU \\
PSWQ & agoraphobia symptoms & BL, Post-T and FU \\
SIAS & Severity of GAD symptoms & BL, Post-T and FU \\
NEO FFI & Severity of SAD symptoms & BL, Post-T and FU \\
EQ-5D & Neuroticism and Extraversion & BL, Post-T and FU \\
OASIS & Health-related quality of life & Post-module \\
ODSIS & Severity of anxiety & Post-module \\
Suicide item & Severity of depression & BL, Post-module, Post-T and FU \\
Expectation of Treatment Scale & Suicidal ideation & BL \\
Opinion of Treatment Scale & Expectation of treatment & Post-T
\end{tabular}

BL, Baseline; Post-T, post-treatment; FU, follow-ups, 3 and 12-month follow-ups; PANAS, Positive and Negative Affect Scale; BDI-II, Beck Depression Inventory-Il; BAI, Beck Anxiety Inventory; OCI-R, Obsessive-Compulsive Inventory-Revised; PDSS-SR, Self-Reported Panic Disorder Severity Scale; PSWQ, Penn State Worry Questionnaire; SIAS, Social Interaction Anxiety Scale; NEO FFI, NEO-Five Factor Inventory; EQ-5D, EuroQoL-5D questionnaire; OASIS, Overall Anxiety Severity and Impairment Scale; ODSIS, Overall Depression Severity and Impairment Scale

compared to verify that there are no significant differences among them at baseline on the outcome measures in order to confirm that they are comparable after randomization. One-way ANOVAs for continuous variables and Chi-squared tests of independence for categorical ones will be used. For the continuous outcome measures on the posttest, the homoscedasticity assumption will be assessed with the Levene test. Where this assumption is met, the usual $F$-test will be applied to compare the posttest means for the three experimental conditions. If the homoscedasticity assumption is not met, the Brown-Forsythe F-test will be applied. Statistically significant $F$-tests will be followed by post hoc comparisons. In particular, the Tukey procedure will be applied where the homoscedasticity assumption is met, and the Games-Howell procedure if this assumption is not met.

The intention-to-treat principle will be used when analyzing primary and secondary post-treatment data and data collected at the 3- and 12-month followups, using mixed-effect models with full information maximum likelihood estimation. This method has been recommended due to its flexibility on repeatedmeasures ANOVAs in handling missing data more appropriately [99]. To complement the results of the ANOVAs and post hoc comparisons, effect sizes will be calculated by using the standardized mean difference proposed by Cohen [62]. These effect sizes will be calculated to assess both within- and between- group changes, all of them based on a pooled standard deviation.

Although per protocol analyses (completers only analysis) suffer from selection bias, they will also be conducted to help to draw conclusions about the maximum treatment efficacy in patients who comply fully with the treatment [100].

When the trial ends, the analytic methodology for the RCT will be reviewed before analyzing the data in order to select the most appropriate analytic procedures.

\section{Discussion}

This study describes a new Internet-based transdiagnostic treatment protocol for patients diagnosed with ED. One of the core aims of this study is to provide data from an RCT to evaluate the efficacy of this protocol in a community sample, compared to a waiting list group. This protocol includes the transdiagnostic cognitivebehavioral principles [17] designed for a wide range of anxiety and depressive disorders to down-regulate NA, and it also incorporates a specific treatment component to address PA. These two ways of delivering the protocol will be tested in order to explore the effect of adding a specific therapeutic component to up-regulate PA.

Moreover, the aim of the present study is to make progress in resolving some of the challenges in the field of mental health [101], specifically in understanding the necessary treatment components to modify clinical symptoms (depression and anxiety) and strengthen 
people's resilience, making them less vulnerable [101, 102]. The potential impact on basic personality dimensions (N/NA/BI) and (E/PA/BA) will also be studied. As Barlow suggests [102], personality dimensions may be malleable over time, and so it can be relevant to study the "malleability" of neuroticism. Likewise, the study will also examine whether it is possible to develop strategies to modify PA (extraversion). There is growing support in the literature for the claim that positive emotions promote flexible and creative thinking and play a fundamental role in the construction of psychological strengths and intellectual and social resources that can be useful in difficult situations in the future [103-106]. Some studies have proposed ways to address both the assessment and treatment of PA regulation from a transdiagnostic perspective [28, 107], but further research is needed on this topic. This study aims to explore the effect of treatment components on increasing wellbeing and PA. If this is confirmed, it would certainly represent an important shift in the research, understanding, assessment, and treatment of ED. To the best of our knowledge, this is the first study of an RCT testing an online transdiagnostic treatment protocol for ED with a specific therapeutic component to directly up-regulate PA.

The data obtained with this study can be compared to results obtained in studies with face-to-face transdiagnostic protocols using benchmarking strategies [33]. In addition, the data will also be compared to transdiagnostic protocols applied over the Internet, but without components for up-regulating PA [15, 108-112].

The objective of establishing action strategies to improve access to EBTs should also be taken into consideration, as well as the goal of providing psychological support to all those in need [5]. Undoubtedly, we are witnessing the beginning of a new era in the field of psychological treatments. They have already gone beyond traditional EBTs, and now it is possible to manage these online protocols with good results [53]. The use of technology and the Internet can help to disseminate and increase the access to these interventions.

Finally, we will test the effectiveness of the application of this Internet-based program with minimal support from the clinician, and the acceptability of this online program in patients from a community sample.

We are aware that this study has limitations. One of the most important is the different number of modules in the two protocols. We tried to control this by giving all the participants equal time and allowing them to use the program as much as they like throughout the whole process. However, in order to test the potential additional impact of the positive psychology component, it seemed necessary to compare it to the traditional transdiagnostic protocol. If the new component is found to have any effect, future research should be carried out to show that this effect is not simply due to the larger number of modules in the protocol.

There are further limitations. For example, dropout rates are expected to be high (around 30\%) [53, 63]. To minimize this problem, human and ICT support will be provided. In addition, another limitation may be recruitment difficulties, to the extent that people still do not have access to the Internet at home, or they have negative attitudes towards Internet interventions.

In summary, this study aims to contribute to the literature on the efficacy of transdiagnostic approaches to ED in general, and it more specifically seeks to explore the possible impact of specific component designed to up-regulate positive affect. Despite its limitations, if positive results are achieved, they will have a clear impact on the design and application of future transdiagnostic treatment protocols for ED, as a way to more effectively address the temperament vulnerabilities, that is, the core aspects of these disorders [102].

\section{Abbreviations}

ADNOS: Anxiety Disorder Not Otherwise Specified; AES: Advanced Encryption Standard; AG: Agoraphobia; ANOVA: Analysis of variance; BAl: Beck Anxiety Inventory; BDI-II: Beck Depression Inventory-II; BL: Baseline; CBT: Cognitive Behavior Therapy; CONSORT: Consolidated Standards Of Reporting Trials; DD: Dysthymia; DSM-IV: Diagnostic and Statistical Manual of mental disorders, fourth text; E/PA/BA: Extraversion/Positive Affect/Behavioral Activation; EBTs: Evidence-based psychological treatments; ED: Emotional disorders; EDBs: Emotion Driven Behaviors; EQ-5D: EuroQol-5D questionnaire; FU: Follow-Ups; GAD: Generalized Anxiety Disorder; ICD-10: International

Classification of Diseases, tenth edition; ICT: Information and Communication Technologies; MDD: Major Depression Disorder; MDNOS: Mood Disorder Not Otherwise Specified; MINI: Mini-International Neuropsychiatric Interview; N/ NA/BI: Neuroticism/Negative Affect/Behavioral Inhibition; NEO-FFI: NEO Five Factor Inventory; OASIS: Overall Anxiety and Impairment Scale;

OCD: Obsessive-compulsive Disorder; OCI-R: Obsessive-Compulsive Inventory-Revised; ODSIS: Overall Depression and Impairment Scale; PANAS: Positive and Negative Affect Schedule; PD: Panic Disorder; PDSSSR: Self-Reported Panic Disorder Severity Scale; POST-T: Post-Treatment; PSWQ: Pen State Worry Questionnaire; RCT: Randomized Controlled Trial; SAD: Social Anxiety Disorder; SI: Social Index; SIAS: Social Interaction Anxiety Inventory; TIBP: Transdiagnostic Internet-Based Protocol; TIBP + PA: Transdiagnostic Internet-Based Protocol + Positive Affect Component; UP: Unified Protocol; VAS: Visual Analogical Scale; WBT: Well-Being Therapy; WL: Waiting List

\section{Acknowledgements}

This study was funded by the Ministry of Economy and Competitiveness (Spain), (Plan Nacional I + D + I. PSI2014-54172-R), and the Institute of Health Carlos III (ISCiii) CIBERobn is an initiative of ISCIII.

\section{Funding}

Funding for the study was provided by grants: CIBER Fisiopatología de la Obesidad y Nutrición-ISCIII CB06/03/0052; Red de Excelencia (PSI2014-56303REDT) PROMOSAM: Research in processes, mechanisms and psychological treatments for mental health promotion, from the Ministry of Economy and Competitiveness (2014); Ministry of Economy and Competitiveness (Spain) (PSI2014-54172-R); and a PhD grant from Ministry of Economy and Competitiveness (FPI-MINECO) (BES-2015-072360). 


\section{Authors' contributions}

AD-G drafted the manuscript with important contributions from $C B$ and AG-P. AD-G, in collaboration with CB and AG-P, designed the study and participated in each of its phases. CB and AG-P designed the traditional version of the transdiagnostic protocol and carried out the Internet-based adaptation with important contributions by RB, AG-R, and JF-A. All authors participated in the review and revision of the manuscript and have approved the final manuscript to be published.

\section{Authors' information}

AD-G is PhD student in Clinical Psychology at Universitat Jaume I in the Department of Basic and Clinical Psychology, and Psychobiology, granted by Ministry of Economy and Competitiveness (FPI-MINECO) (BES-2015-072360). AD-G is also member of LabPsiTec (Laboratory of Psychology and Technology) (www.labpsitec.com) at Universitat Jaume I. AG-R and JF-A are PhD students in Clinical Psychology and members of LabPsiTec. AG-P is a professor of Psychopathology at Universitat Jaume I in the Department of Basic and Clinical Psychology, and Psychobiology. RMB is full professor at Universitat de Valencia, in the Department of Personality, Evaluation and Psychological Treatments, and she is member of LabPsiTec. CB is full professor of Clinical Psychology at Universitat Jaume I in the Department of Basic and Clinical Psychology, and Psychobiology. She is also the Director of LabPsiTec and Head of a CIBER (Centre for Network Biomedical Research) group in the area of Biomedicine and Health Sciences (www.ciberisciii.es). CB is also director of the Doctoral Program in Psychology at Universitat Jaume I.

\section{Competing interests}

The authors declare that they have no competing interests.

\section{Consent for publication}

"Not applicable" in this section.

\section{Ethics approval and consent to participate}

The study follows the guidelines of the Declaration Helsinki and existing guidelines in Spain and the European Union for the protection of patients in clinical trials. All participants interested in participating signed an informed consent form. The study has been approved by the Ethics Committee of Universitat Jaume I (Castellón, Spain) (October, 2015). The trial was registered at ClinicalTrial.gov as NCT02578758.

\section{Publisher's Note}

Springer Nature remains neutral with regard to jurisdictional claims in published maps and institutional affiliations.

\section{Author details}

${ }^{1}$ Universitat Jaume I, Castellón, Spain. Universidad de Valencia, Valencia, Spain. ${ }^{3}$ CIBER Fisiopatología Obesidad y Nutrición (CIBERObn), Instituto Salud Carlos III, Madrid, Spain.

Received: 22 March 2017 Accepted: 1 April 2017

Published online: 20 April 2017

\section{References}

1. Kessler RC, Berglund P, Demler O, Jin R, Merikangas KR, Walters EE. Lifetime prevalence and age-of-onset distributions of DSM-IV disorders in the National Comorbidity Survey Replication. Arch Gen Psychiatry. 2005;62(6):593-602

2. McLean CP, Asnaani A, Litz BT, Hofmann SG. Gender differences in anxiety disorders: prevalence, course of illness, comorbidity and burden of illness. J Psychiatr Res. 2011;45(8):1027-35.

3. Kazdin AE, Rabbitt SM. Novel models for delivering mental health services and reducing the burdens of mental illness. Clin Psychol Sci. 2013;1(2):170-91.

4. Nathan PE, Gorman JM. A guide to treatments that work. 4th ed. New York: Oxford University Press; 2016.

5. Kazdin AE. Clinical dysfunction and psychosocial interventions: the interplay of research, Methods, and conceptualization of challenges. Annu Rev Clin Psychol. 2015;11:25-52.

6. Kazdin $A E$, Blase SL. Rebooting psychotherapy research and practice to reduce the burden of mental illness. Perspect Psychol Sci. 2011;6(1):21-37.
7. Bebbington PE, Meltzer $H$, Brugha TS, Farrell M, Jenkins R, Ceresa $C$, et al. Unequal access and unmet need: neurotic disorders and the use of primary care services. Psychol Med. 2000;30(6):1359-67.

8. Emmelkamp PMG, David D, Beckers T, Muris P, Cuijpers P, Lutz W, et al. Advancing psychotherapy and evidence-based psychological interventions. Int J Methods Psychiatr Res. 2014;23(1):58-91.

9. Ellard KK, Fairholme CP, Boisseau CL, Farchione TJ, Barlow DH. Unified protocol for the Transdiagnostic treatment of emotional disorders: protocol development and initial outcome data. Cogn Behav Pract. 2010;17(1):88-101.

10. McHugh RK, Barlow DH. The dissemination and implementation of evidence-based psychological treatments. A review of current efforts. Am Psychol. 2010;65(2):73-84.

11. Haro JM, Ayuso-Mateos JL, Bitter I, Demotes-Mainard J, Leboyer M, Lewis SW, et al. ROAMER: roadmap for mental health research in Europe. Int J Methods Psychiatr Res. 2014;23(1):1-14.

12. Kazdin AE. Evidence-based psychotherapies I: qualifiers and limitations in what we know. South African J Psychol. 2014;44(4):381-403.

13. Antony MM, Stein MB. Oxford handbook of anxiety and related disorders. New York: Oxford University Press; 2009.

14. Barlow DH. Anxiety and its disorders: the nature and treatment of anxiety and panic. 2nd ed. New York: Guilford Press; 2002.

15. Norton PJ, Price EC. A meta-analytic review of adult cognitive-behavioral treatment outcome across the anxiety disorders. J Nerv Ment Dis. 2007; 195(6):521-31.

16. Smits JAJ, Hofmann SG. A meta-analytic review of the effects of psychotherapy control conditions for anxiety disorders. Psychol Med. 2009;39(2):229.

17. Barlow DH, Allen LB, Choate ML. Toward a unified treatment for emotional disorders. Behav Ther. 2004;35:205-30.

18. Brown TA, Barlow DH. A proposal for a dimensional classification system based on the shared features of the DSM-IV anxiety and mood disorders: implications for assessment and treatment. Psychol Assess. 2009;21(3):256-71.

19. Brown TA. Temporal course and structural relationships among dimensions of temperament and DSM-IV anxiety and mood disorder constructs. J Abnorm Psychol. 2007;116(2):313-28.

20. Campbell-Sills L, Barlow DH, Brown TA, Hofmann SG. Effects of suppression and acceptance on emotional responses of individuals with anxiety and mood disorders. Behav Res Ther. 2006:44:1251-63.

21. Mennin DS, Heimberg RG, Turk CL, Fresco DM. Preliminary evidence for an emotion dysregulation model of generalized anxiety disorder. Behav Res Ther. 2005:43:1281-310.

22. Weiss NH, Tull MT, Davis LT, Dehon EE, Fulton JJ, Gratz KL. Examining the Association between emotion regulation difficulties and probable posttraumatic stress disorder within a sample of African Americans. Cogn Behav Ther. 2012;41(1):5-14. http://dx.doi.org/10.1080/16506073. 2011.621970

23. Roemer L, Salters K, Raffa SD, Orsillo SM. Fear and avoidance of internal experiences in GAD: preliminary tests of a conceptual model. Cognit Ther Res. 2005;29(1):71-88. http://dx.doi.org/10.1007/s10608-005-1650-2.

24. Aldao A, Nolen-Hoeksema S, Schweizer S. Emotion-regulation strategies across psychopathology: a meta-analytic review. Clin Psychol Rev. 2010; 30(2):217-37. http://dx.doi.org/10.1016/j.cpr.2009.11.004.

25. Boswell JF, Thompson-Hollands J, Farchione TJ, Barlow DH. Intolerance of uncertainty: a common factor in the treatment of emotional disorders. J Clin Psychol. 2013;69(6):630-45. http://dx.doi.org/10.1002/jclp.21965.

26. Barlow DH, Ellard KK, Sauer-Zavala S, Bullis JR, Carl JR. The origins of neuroticism. Perspect Psychol Sci. 2014;9(5):481-96. http://dx.doi.org/10. $1177 / 1745691614544528$.

27. Kotov R, Gamez W, Schmidt F, Watson D. Linking "big" personality traits to anxiety, depressive, and substance use disorders: a meta-analysis. Psychol Bull. 2010;136(5):768-821. http://dx.doi.org/10.1037/a0020327.

28. Carl JR, Soskin DP, Kerns C, Barlow DH. Positive emotion regulation in emotional disorders: a theoretical review. Clin Psychol Rev. 2013;33(3): 343-60. http://dx.doi.org/10.1016/j.cpr.2013.01.003.

29. Lopez SJ, Snyder CR. The Oxford handbook of positive psychology. Oxford library of psychology; 2009.

30. Gilbert KE, Nolen-Hoeksema S, Gruber J. Positive emotion dysregulation across mood disorders: how amplifying versus dampening predicts emotional reactivity and illness course. Behav Res Ther. 2013;51(11):736-41. http://dx.doi. org/10.1016/j.brat.2013.08.004.

31. Fredrickson BL. The role of positive emotions in positive psychology: the broaden-and-build theory of positive emotions. Am Psychol. 2001;56(3):218-26. 
32. Allen LB, McHugh RK, Barlow DH. Emotional disorders: A unified protocol. New York: Guilford Press; 2008.

33. Farchione TJ, Fairholme CP, Ellard KK, Boisseau CL, Thompson-Hollands J, Carl JR, et al. Unified protocol for Transdiagnostic treatment of emotional disorders: a randomized controlled trial. Behav Ther. 2012;43(3):666-78.

34. Bullis JR, Fortune MR, Farchione TJ, Barlow DH. A preliminary investigation of the long-term outcome of the unified protocol for Transdiagnostic treatment of emotional disorders. Compr Psychiatry. 2014;55(8):1920-7.

35. Carl JR, Gallagher MW, Sauer-Zavala SE, Bentley KH, Barlow DH. A preliminary investigation of the effects of the unified protocol on temperament. Compr Psychiatry. 2014;55(6):1426-34. http://dx.doi.org/10. 1016/j.comppsych.2014.04.015.

36. Clark DA, Taylor S. The Transdiagnostic perspective on cognitive-behavioral therapy for anxiety and depression: new wine for old wineskins? J Cogn Psychother. 2009;23(1):60-6.

37. Reinholt N, Krogh J. Efficacy of Transdiagnostic cognitive behaviour therapy for anxiety disorders: a systematic review and meta-analysis of published outcome studies. Cogn Behav Ther. 2014;43(3):171-84.

38. Newby JM, McKinnon A, Kuyken W, Gilbody S, Dalgleish T. Systematic review and meta-analysis of transdiagnostic psychological treatments for anxiety and depressive disorders in adulthood. Clin Psychol Rev. 2015;40:91-110. http://dx. doi.org/10.1016/j.cpr.2015.06.002.

39. Newby JM, Twomey C, Yuan Li SS, Andrews G. Transdiagnostic computerised cognitive behavioural therapy for depression and anxiety: a systematic review and meta-analysis. J Affect Disord. 2016;199:30-41.

40. Păsărelu $C R$, Andersson $G$, Bergman Nordgren L, Dobrean A. Internetdelivered transdiagnostic and tailored cognitive behavioral therapy for anxiety and depression: a systematic review and meta-analysis of randomized controlled trials. Cogn Behav Ther. 2017;46(1):1-28. http://dx. doi.org/10.1080/16506073.2016.1231219.

41. WORLD HEALTH ORGANIZATION. Promoting mental health: concepts, emerging evidence, practice: summary report. 2004.

42. Southwick SM, Charney DS. Resilience. Cambridge: Cambridge University Press; 2012.

43. Keyes CLM, Dhingra SS, Simoes EJ. Change in level of positive mental health as a predictor of future risk of mental illness. Am J Public Health. 2010; 100(12):2366-71. http://dx.doi.org/10.2105/AJPH.2010.192245.

44. Wood AM, Joseph S. The absence of positive psychological (eudemonic) well-being as a risk factor for depression: a ten year cohort study. J Affect Disord. 2009:122:213-7.

45. Bolier L, Haverman M, Westerhof GJ, Riper H, Smit F, Bohlmeijer E, et al. Positive psychology interventions: a meta-analysis of randomized controlled studies. BMC Public Health. 2013;13(1):119. http://dx.doi.org/10. 1186/1471-2458-13-119.

46. Roepke AM, Seligman MEP. Depression and prospection. Br J Clin Psychol. 2016;55(1):23-48. http://dx.doi.org/10.1111/bjc.12087.

47. Vos SPF, Huibers MJH, Diels L, Arntz A. A randomized clinical trial of cognitive behavioral therapy and interpersonal psychotherapy for panic disorder with agoraphobia. Psychol Med. 2012;42(12):2661-72. https://doi.org/10.1017/ S0033291712000876.

48. Botella C, Gallego MJ, Garcia-Palacios A, Baños RM, Quero S, Alcañiz M. The acceptability of an internet-based self-help treatment for fear of public speaking. Br J Guid Counc. 2009;37(3):297-311. http://dx.doi.org/10.1080/ 03069880902957023.

49. Kazdin AE. Technology-based interventions and reducing the burdens of mental illness: perspectives and comments on the special series. Cogn Behav Pract. 2015;22(3):359-66. http://dx.doi.org/10.1016/j.cbpra.2015.04.004.

50. Andersson G. Internet-delivered psychological treatments. Annu Rev Clin Psychol. 2016;12(1):157-79. http://dx.doi.org/10.1146/annurev-clinpsy021815-093006.

51. Andersson G. Using the internet to provide cognitive behaviour therapy. Behav Res Ther. 2009:47(3):175-80. http://dx.doi.org/10.1016/j.brat.2009.01.010.

52. Andersson G, Cuijpers P. Internet-based and other computerized psychological treatments for adult depression: a meta-analysis. Cogn Behav Ther. 2009;38(4):196-205. http://dx.doi.org/10.1080/16506070903318960.

53. Andrews G, Cuijpers P, Craske MG, McEvoy P, Titov N. Computer therapy for the anxiety and depressive disorders is effective, acceptable and practical health care: a meta-analysis. PLoS One. 2010;5(10):e13196. http://dx.doi.org/ 10.1371/journal.pone.0013196.

54. Cuijpers P, Donker T, Johansson R, Mohr DC, van Straten A, Andersson G. Self-guided psychological treatment for depressive symptoms: a meta- analysis. PLoS One. 2011;6(6):e21274. http://dx.doi.org/10.1371/journal.pone. 0021274.

55. Cuijpers $P$, van Straten A, Warmerdam L, Andersson G. Psychotherapy versus the combination of psychotherapy and pharmacotherapy in the treatment of depression: a meta-analysis. Depress Anxiety. 2009;26(3):279-88.

56. American Psychological Association. Diagnostic and statistical manual of mental disorders. 4th ed. Rev ed. Washington: American Psychiatric Association; 2000.

57. Moher D, Schulz KF, Altman DG. The CONSORT statement: revised recommendations for improving the quality of reports of parallel group randomized trials. J Am Podiatr Med Assoc. 2001;91(8):437-42.

58. Moher D, Hopewell S, Schulz KF, Montori V, Gøtzsche PC, Devereaux PJ, et al. CONSORT 2010 explanation and elaboration: updated guidelines for reporting parallel group randomised trials. J Clin Epidemiol. 2010;63(8):e1-37.

59. Eysenbach G. CONSORT-EHEALTH: improving and standardizing evaluation reports of web-based and mobile health interventions. J Med Internet Res. 2011;13(4):e126.

60. Chan A-W, Tetzlaff JM, Altman DG, Laupacis A, Gøtzsche PC, Krleža-Jerić K, et al. SPIRIT 2013 statement: defining standard protocol items for clinical trials. Ann Intern Med. 2013;158(3):200.

61. Chan A-W, Tetzlaff JM, Gøtzsche PC, Altman DG, Mann H, Berlin JA, et al. SPIRIT 2013 explanation and elaboration: guidance for protocols of clinical trials. BMJ. 2013;346:e7586.

62. Cohen J. Statistical power analysis for the behavioral sciences. 2nd ed. L. Erlbaum Associates: Hillsdale; 1988.

63. Van Ballegooijen W, Cuijpers P, van Straten A, Karyotaki E, Andersson G, Smit JH, et al. Adherence to internet-based and face-to-face cognitive Behavioural therapy for depression: a meta-analysis. PLoS One. 2014;9(7): e100674. http://dx.plos.org/10.1371/journal.pone.0100674.

64. Faul F, Erdfelder E, Lang A-G, Buchner A. G*power 3: a flexible statistical power analysis program for the social, behavioral, and biomedical sciences. Behav Res Methods. 2007;39(2):175-91. http://dx.doi.org/10.3758/ BF03193146.

65. Altman DG, Schulz KF, Moher D, Egger M, Davidoff F, Elbourne D, et al. The revised CONSORT statement for reporting randomized trials: explanation and elaboration. Ann Intern Med. 2001;134(8):663-94.

66. Linehan M. Cognitive-behavioral treatment of borderline personality disorder. New York: Guilford Press; 1993.

67. Sin NL, Lyubomirsky S. Enhancing well-being and alleviating depressive symptoms with positive psychology interventions: a practice-friendly metaanalysis. J Clin Psychol Sess J Clin Psychol Sess. 2009;65(655):467-87.

68. Lejuez CW, Hopko DR, Hopko SD. A brief behavioral activation treatment for depression: treatment manual. Behav Modif. 2001;25(2):255-86.

69. Seligman MEP, Csikszentmihalyi M. Positive psychology: an introduction. Am Psychol. 2000:55(1):5-14.

70. Fava GA. Well-being therapy: conceptual and technical issues. Psychother Psychosom. 1999;68(4):171-9.

71. Fava GA, Ruini C. Development and characteristics of a well-being enhancing psychotherapeutic strategy: well-being therapy. J Behav Ther Exp Psychiatry. 2003;34(1):45-63.

72. Sheehan DV, Lecrubier Y, Sheehan KH, Amorim P, Janavs J, Weiller E, et al. The Mini-International Neuropsychiatric interview (M.I.I.I.): the development and validation of a structured diagnostic psychiatric interview for DSM-IV and ICD-10. J Clin Psychiatry. 1998;59(20):22-33.

73. Lecrubier Y, Sheehan D, Weiller E, Amorim P, Bonora I, Harnett Sheehan K, et al. The MINI International Neuropsychiatric interview (MINI). A short diagnostic structured interview: reliability and validity according to the CIDI. Eur Psychiatry. 1997;12(5):224-31.

74. Ferrando L, Franco-A L, Soto M, Bobes J, Soto O, Franco L, et al. M.I.N.I. Mini International Neuropsychiatric interview. Spanish version 5.0.0. DSM-IV. Madrid: Instituto IAP; 1998.

75. Beck AT, Steer RA, Brown GK. Manual for the Beck depression Inventory-II. San Antonio: The Psychological Corporation; 1990.

76. Sanz J, Navarro ME, Vázquez C. Adaptación española del Inventario para la Depresión de Beck-II (BDI-II): propiedades psicométricas en estudiantes universitarios. Anal Mod Cond. 2003;29(124):239-88.

77. Beck AT, Steer RA. Beck anxiety Inventory manual. San Antonio: The Psychological Corporation; 1993.

78. Magán I, Sanz J, García-Vera MP. Psychometric properties of a Spanish version of the Beck anxiety Inventory (BAI) in general population. Span J Psychol. 2008;11(2):626-40. 
79. Watson D, Clark LA, Tellegen A. Development and validation of brief measures of positive and negative affect: the PANAS scales. J Pers Soc Psychol. 1988;54(6):1063-70.

80. Sandín B, Chorot P, Lostao L, Joiner TE, Santed MA, Valiente RM. Escalas PANAS de afecto positivo y negativo: Validacion factorial y convergencia transcultural. Psicothema. 1999;11(1):37-51.

81. Foa EB, Huppert JD, Leiberg S, Langner R, Kichic R, Hajcak G, et al. The obsessive-compulsive Inventory: development and validation of a short version. Psychol Assess. 2002;14(4):485-96.

82. Fullana MA, Tortella-Feliu M, Caseras X, Andión O, Torrubia R, Mataix-Cols D. Psychometric properties of the Spanish version of the obsessivecompulsive Inventory-revised in a non-clinical sample. J Anxiety Disord. 2005;19(8):893-903.

83. Houck PR, Spiegel DA, Shear MK, Rucci P. Reliability of the self-report version of the panic disorder severity scale. Depress Anxiety. 2002;15(4): 183-5.

84. Meyer TJ, Miller ML, Metzger RL, Borkovec TD. Development and validation of the Penn State worry questionnaire. Behav Res Ther. 1990;28(6):487-95.

85. Sandín B, Chorot P, Valiente RM, Lostao L. Validación española del cuestionario de preocupación PSWQ: Estructura factorial y propiedades psicométricas. Rev Psicopatol Psicol Clin. 2009;14(2):107-22.

86. Mattick RP, Clarke JC. Development and validation of measures of social phobia scrutiny fear and social interaction anxiety. Behav Res Ther. 1998; 36(4):455-70. http://dx.doi.org/10.1016/S0005-7967(97)10031-6.

87. Zubeidat I, Salinas JM, Sierra JC, Fernández-Parra A. Psychometric properties of the social interaction anxiety scale and separation criterion between Spanish youths with and without subtypes of social anxiety. J Anxiety Disord. 2007;21(5):603-24. http://dx.doi.org/10.1016/j.janxdis.2006.09.010.

88. Costa PT, McCrae RR. Normal personality assessment in clinical practice: the NEO personality Inventory. Psychol Assess. 1992;4(1):5-13.

89. Robins RW, Fraley RC, Roberts BW, Trzesniewski KH. A longitudinal study of personality change in young adulthood. J Pers. 2001;69(4):617-40. http://dx. doi.org/10.1111/1467-6494.694157.

90. Aluja A, García O, Rossier J, García LF. Comparison of the NEO-FFl, the NEOFFI-R and an alternative short version of the NEO-PI-R (NEO-60) in Swiss and Spanish samples. Pers Individ Dif. 2005;38(3):591-604.

91. Badia X, Roset M, Montserrat S, Herdman M, Segura A. The Spanish version of EuroQol: a description and its applications. European quality of life scale. Med Clin. 1998;112:79-85.

92. Bentley KH, Gallagher MW, Carl JR, Barlow DH. Development and validation of the Overall depression severity and impairment scale. Psychol Assess. 2014;26(3):815-30. http://dx.doi.org/10.1037/a0036216.

93. Norman SB, Campbell-Sills L, Hitchcock CA, Sullivan S, Rochlin A, Wilkins KC, et al. Psychometrics of a brief measure of anxiety to detect severity and impairment: the Overall anxiety severity and impairment scale (OASIS). J Psychiatr Res. 2011;45(2):262-8. http://dx.doi.org/10.1016/j.jpsychires.2010. 06.011.

94. Mira A, González A, Botella C, Bretón-López J, García-Palacios A, Riera A, et al. Psychometric properties of the Overall Anxiety Severity and Impairment Scale in Spanish sample. Clinical and general populations. Poster session presented at: ABCT Convention. Chicago: 49th Annual Conference; 2015. Available in: https://goo.gl/8KdNJK.

95. Gonzalez-Robles A, Mira A, Breton-Lopez J, Riera A, Raya D, Baños RM, et al. Psychometric properties of the Overall Depression Severity and Impairment Scale in Spanish sample. General and Clinical populations. Poster session presented at: ESRII. Warsaw: 3rd Conference; 2015. Available in: https://goo. gl/97q0LH.

96. Borkovec TD, Nau SD. Credibility of analogue therapy rationales. J Behav Ther Exp Psychiatry. 1972;3(4):257-60. http://dx.doi.org/10.1016/00057916(72)90045-6.

97. Botella C, García-Palacios A, Villa H, Baños RM, Quero S, Alcañiz M, et al. Virtual reality exposure in the treatment of panic disorder and agoraphobia: a controlled study. Clin Psychol Psychother Clin Psychol Psychother. 2007:14:164-75.

98. Schulz KF, Altman DG, Moher D, Jüni P, Altman D, Egger M, et al. CONSORT 2010 statement: updated guidelines for reporting parallel group randomised trials. BMC Med. 2010;8(1):18. http://dx.doi.org/10.1186/1741-7015-8-18.

99. Gueorguieva R, Krystal JH. Move over ANOVA: progress in analyzing repeated-measures data and its reflection in papers published in the archives of general Psychiatry. Arch Gen Psychiatry. 2004;61(3):310. http://dx. doi.org/10.1001/archpsyc.61.3.310.
100. Wright CC, Sim J. Intention-to-treat approach to data from randomized controlled trials: a sensitivity analysis. J Clin Epidemiol. 2003;56(9):833-42.

101. Collins PY, Patel V, Joestl SS, March D, Insel TR, Daar AS, et al. Grand challenges in global mental health. Nature. 2011:475(7354):27-30. http://dx.doi.org/10. 1038/475027a.

102. Barlow DH, Sauer-Zavala S, Carl JR, Bullis JR, Ellard KK. The nature, diagnosis, and treatment of neuroticism: back to the future. Clin Psychol Sci. 2013;2(3):344-65.

103. Algoe SB, Fredrickson BL. Emotional fitness and the movement of affective science from lab to field. Am Psychol. 2011;66(1):35-42. http://dx.doi.org/10. 1037/a0021720

104. Catalino LI, Fredrickson BL. A Tuesday in the life of a flourisher: the role of positive emotional reactivity in optimal mental health. Emotion. 2011;11(4):938-50 http://dx.doi.org/10.1037/a0024889.

105. McMahan EA, Renken MD. Eudaimonic conceptions of well-being, meaning in life, and self-reported well-being: initial test of a mediational model. Pers Individ Dif. 2011;51(5):589-94. http://dx.doi.org/10.1016/j.paid.2011.05.020.

106. Wood AM, Froh JJ, Geraghty AWA. Gratitude and well-being: a review and theoretical integration. Clin Psychol Rev. 2011;30(7):890-905.

107. Carl JR, Fairholme CP, Gallagher MW, Thompson-Hollands J, Barlow DH. The effects of anxiety and depressive symptoms on daily positive emotion regulation. J Psychopathol Behav Assess. 2014;36(2):224-36. http://dx.doi. org/10.1007/s10862-013-9387-9.

108. Mcevoy PM, Nathan P, Norton PJ. Efficacy of Transdiagnostic treatments: a review of published outcome studies and future research directions. J Cogn Psychother. 2009;23(1):20-33.

109. Olatunji BO, Tart CD, Ciesielski BG, McGrath PB, Smits JAJ. Specificity of disgust vulnerability in the distinction and treatment of OCD. J Psychiatr Res. 2011;45(9):1236-42. http://dx.doi.org/10.1016/j.jpsychires.2011.01.018.

110. Richards D, Richardson T. Computer-based psychological treatments for depression: a systematic review and meta-analysis. Clin Psychol Rev. 2012;32(4):329-42. http://dx.doi.org/10.1016/j.cpr.2012.02.004.

111. Titov N, Dear BF, Johnston L, Lorian C, Zou J, Wootton B, et al. Improving adherence and clinical outcomes in self- guided internet treatment for anxiety and depression: randomised controlled trial. PLoS One. 2013;8(7):e62873. http://dx.doi.org/10.1371/journal.pone.0062873.

112. Titov N, Dear BF, Staples LG, Terides MD, Karin E, Sheehan J, et al. Disorderspecific versus transdiagnostic and clinician-guided versus self-guided treatment for major depressive disorder and comorbid anxiety disorders: a randomized controlled trial. J Anxiety Disord. 2015;35:88-102.

\section{Submit your next manuscript to BioMed Central and we will help you at every step:}

- We accept pre-submission inquiries

- Our selector tool helps you to find the most relevant journal

- We provide round the clock customer support

- Convenient online submission

- Thorough peer review

- Inclusion in PubMed and all major indexing services

- Maximum visibility for your research

Submit your manuscript at www.biomedcentral.com/submit
Biomed Central 